\title{
Off-diagonal properties of the Feynman propagator and the Green function for a bare Coulomb field
}

\author{
I. A. Howard ${ }^{1}$ and N. H. March ${ }^{1,2}$ \\ ${ }^{1}$ Department of Physics, University of Antwerp, Groenenborgerlaan 171, B-2020 Antwerp, Belgium \\ ${ }^{2}$ Oxford University, Oxford, United Kingdom
}

(Received 14 January 2004; published 13 April 2004)

\begin{abstract}
Blinder [Phys. Rev. A, 43, 13 (1991)] has given an explicit diagonal form for the nonrelativistic Coulomb propagator. His result is an infinite series involving Whittaker, Laguerre, Hermite, and error functions. However, he notes that at the nucleus, only error functions remain in the infinite series. Here, an off-diagonal generalization of Blinder's result at the nucleus is constructed. By relating this result to the Green function, it is then shown that one is led in this latter representation to an explicit sum of Blinder's infinite series. Finally, contact is established between the present off-diagonal study and the recent work of Chibisov et al. [Phys. Rev. Lett. 84, 451 (2000)] on the Coulomb Green function.
\end{abstract}

DOI: 10.1103/PhysRevA.69.044703

PACS number(s): $34.10 .+\mathrm{x}, 31.15 .-\mathrm{p}$

Though numerous studies now exist of the nonrelativistic Feynman propagator for a bare Coulomb field, the most explicit result known to the present writers is that contained in the work of Blinder [1], where earlier references may be found. Because the first part of the present Brief Report is concerned with a direct generalization of Blinder's work, we shall adopt his notation below. Thus, we define the Feynman propagator in terms of Coulomb wave functions $\psi_{n}(\mathbf{r})$ with corresponding energies $E_{n}$ as

$$
K\left(\mathbf{r}_{1}, \mathbf{r}_{2}, t\right)=\sum_{n} \psi_{n}\left(\mathbf{r}_{1}\right) \psi_{n}^{*}\left(\mathbf{r}_{2}\right) \exp \left(-i E_{n} t\right)
$$

For compactness, Blinder introduces the variable $\beta=i t$ and then the propagator has the form of a statistical mechanical density matrix [with $\beta$ standing for $\left(k_{B} T\right)^{-1}$.

Blinder's most explicit result is for the diagonal form $K(\mathbf{r}, \mathbf{r}, \beta)$. This is given in his Eq. (31) as an infinite series involving Whittaker, Laguerre, Hermite, and error functions. However, at the nucleus $\mathbf{r}=\mathbf{0}$, he notes that $K(0,0, \beta)$ is given explicitly by [his Eq. (32)]

$$
\begin{aligned}
K(0,0, \beta)= & (2 \pi \beta)^{-3 / 2}+\frac{Z}{2 \pi \beta}+\frac{1}{\pi^{3 / 2}(2 \beta)^{1 / 2}} \sum_{n=1}^{\infty} Z_{n}^{2} \\
& +\frac{1}{2 \pi} \sum_{n=1}^{\infty} Z_{n}^{3} \exp \left(\beta Z_{n}^{2} / 2\right) \operatorname{erfc}\left(-Z_{n} \sqrt{\beta / 2}\right): Z_{n}=Z / n
\end{aligned}
$$

Having referred to the statistical mechanical density matrix with $\beta \equiv\left(k_{B} T\right)^{-1}$ above, we next note that March and Murray $[2,3]$ gave a quite explicit perturbation expansion in the potential energy $V(\mathbf{r})$, throughout the present Brief Report specialized to the Coulomb form

$$
V(\mathbf{r})=V(|\mathbf{r}|)=-Z / r
$$

of $K\left(\mathbf{r}_{1}, \mathbf{r}_{2}, \beta\right)$, with plane waves constituting the unperturbed form. Here, we shall be able to make some analytical progress with the off-diagonal generalization $K(\mathbf{r}, 0, \beta)$ of
Blinder's form (2). Following March and Murray [2], we can write from their Eq. (3.1),

$$
K(r, 0, \beta)=(2 \pi \beta)^{-3 / 2} \exp \left(-r^{2} / 2 \beta\right)+O(Z)
$$

where evidently at $r=0$ the $O(Z)$ contribution in Eq. (4) is $Z / 2 \pi \beta$ from Blinder's result in Eq. (2) above. Denoting the $O(Z)$ term in $K(r, 0, \beta)$ by $K_{1}(r, 0, \beta)$, the March-Murray result [3] is then (see also March, Young, and Sampanthar [4])

$$
\begin{aligned}
K_{1}(r, 0, \beta)= & (2 \pi \beta)^{-3 / 2} \frac{Z}{2 \pi} \int \frac{r_{1}+\left|\mathbf{r}-\mathbf{r}_{1}\right|}{r_{1}^{2}\left|\mathbf{r}-\mathbf{r}_{1}\right|} \\
& \times \exp \left(-\frac{1}{2 \beta}\left(r_{1}+\left|\mathbf{r}-\mathbf{r}_{1}\right|\right)^{2}\right) d \mathbf{r}_{1},
\end{aligned}
$$

which can be evaluated to give

$$
K_{1}(r, 0, \beta)=-\frac{Z}{2^{3 / 2} \sqrt{\pi \beta}} \frac{\exp \left(-r^{2} / 2 \beta\right)}{r}\left[i \operatorname{erf}\left(\frac{i r}{\sqrt{2 \beta}}\right)\right] \text {. }
$$

This expression (6) is plotted as a function of $r$ for the two values of $\beta$ recorded in Fig. 1. We have confirmed that, in spite of its superficial appearance, Eq. (6) does indeed exactly represent the purely real quantity $K_{1}(r, 0, \beta)$ given by Eq. (5). Higher-order terms are immediately available from the March-Murray perturbation theory: it will be convenient below to formally subsume these into a "remainder" $R(r, 0, \beta)$ defined by

$$
K(r, 0, \beta)-(2 \pi \beta)^{-3 / 2} \exp \left(-r^{2} / 2 \beta\right)-K_{1}(r, 0, \beta)=R(r, 0, \beta) .
$$

$R(r, 0, \beta)$, of course, is intended thereby to represent the summation of all terms of $O\left(Z^{2}\right)$ and higher, and thus we can write by comparison of Eqs. (2) and (7) that 


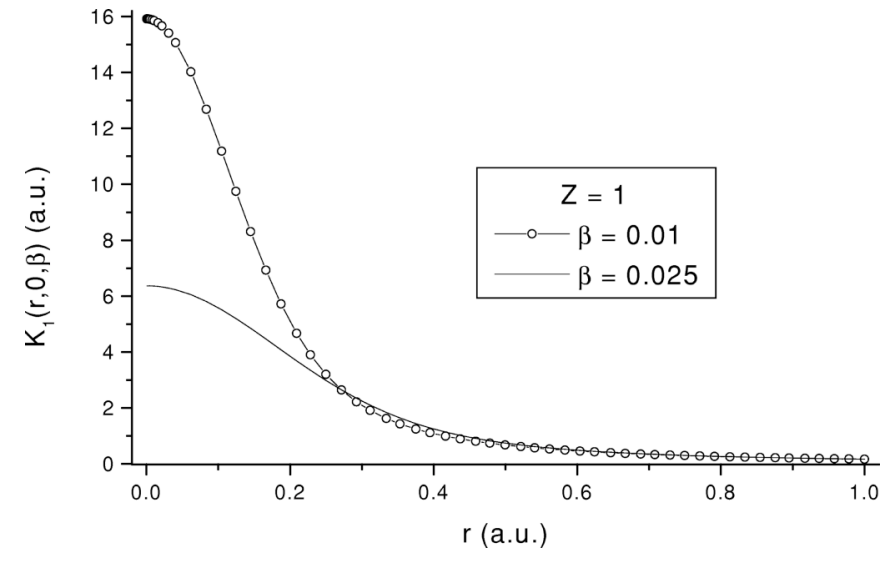

FIG. 1. The $O(Z)$ term [Eq. (6)] $K_{1}(r, 0, \beta)$ in the March-Murray expansion for the propagator $K(r, 0, \beta)$ for the Coulomb potential. Results are shown here for $Z=1$ and for the values $\beta=0.01$ and 0.025

$$
\begin{aligned}
R(0,0, \beta)= & \frac{1}{\pi^{3 / 2}(2 \beta)^{1 / 2}} \sum_{n=1}^{\infty} Z_{n}^{2}+\frac{1}{2 \pi} \sum_{n=1}^{\infty} Z_{n}^{3} \exp \left(\beta Z_{n}^{2} / 2\right) \\
& \times \operatorname{erfc}\left(-Z_{n} \sqrt{\beta / 2}\right)
\end{aligned}
$$

We turn next to consider the Green function $G\left(\mathbf{r}_{1}, \mathbf{r}_{2}, E\right)$, which we again define, following Blinder's Eq. (6), as

$$
G\left(\mathbf{r}_{1}, \mathbf{r}_{2}, E\right)=\sum_{n} \frac{\psi_{n}\left(\mathbf{r}_{1}\right) \psi_{n}^{*}\left(\mathbf{r}_{2}\right)}{E-E_{n}+i \varepsilon}
$$

We next note the important connection, to be exploited below, that the Laplace transform $(\equiv \mathcal{L})$ of $K\left(\mathbf{r}_{1}, \mathbf{r}_{2}, \beta\right)$ with respect to $\beta(\rightarrow E)$ is related to the Green function by

$$
\mathcal{L} K\left(\mathbf{r}_{1}, \mathbf{r}_{2}, \beta\right)=G\left(\mathbf{r}_{1}, \mathbf{r}_{2},-E\right) .
$$

As the most elementary example, take the plane wave term in Eq. (4), and one finds from Laplace transform tables [5]

$$
\mathcal{L}\left[(2 \pi \beta)^{-3 / 2} \exp \left(-r^{2} / 2 \beta\right)\right]=\frac{\exp (-\sqrt{2 E} r)}{r} .
$$

But the corresponding free-electron Green function $G_{0}(r, 0, E)$ is given by the outgoing spherical wave

$$
G_{0}(r, 0, E)=\frac{\exp (i k r)}{r}, \quad \frac{k^{2}}{2} \equiv E,
$$

and hence

$$
\mathcal{L}\left[(2 \pi \beta)^{-3 / 2} \exp \left(-r^{2} / 2 \beta\right)\right]=G_{0}(r, 0,-E) .
$$

The merit of Eq. (10), with the special case (13) corresponding to $Z=0$ in the present Coulomb study, is that it can be used to sum the infinite series of terms in Eq. (8), as will now be demonstrated. While, as is immediately clear from Eq. (11), the Laplace transform of $K(\mathbf{r}, 0, \beta)$ in Eq. (7) diverges as $r \rightarrow 0$, the quantity $R(r, 0, \beta)$ defined in Eq. (7) has a finite Laplace transform as $r \rightarrow 0$. Thus, we can immediately focus on the Blinder form $R(0,0, \beta)$ in Eq. (8). Again, using tables of Laplace transforms, we find

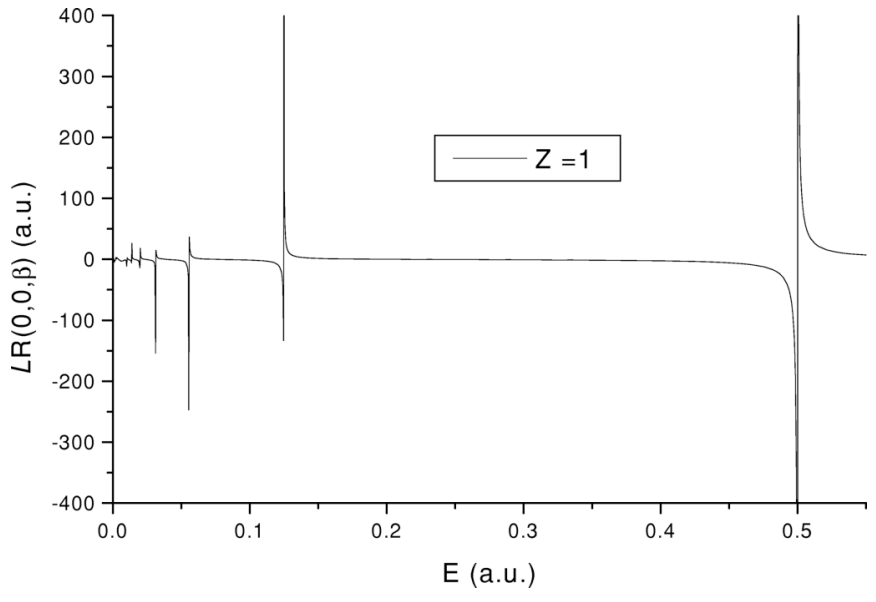

FIG. 2. Equation (14) for the Laplace transform of the remainder term $R(0,0, \beta)$. The case $Z=1$ is shown.

$$
\mathcal{L} R(0,0, \beta)=-\frac{Z \gamma}{\pi}-\frac{Z}{\pi} \Psi\left(1-\frac{Z}{\sqrt{2 E}}\right),
$$

where $\gamma \simeq 0.577$ is Euler's constant while $\Psi$ denotes the digamma function

$$
\Psi(x)=\frac{d}{d x} \ln \Gamma(x) .
$$

Though we have not presently succeeded in getting an explicit off-diagonal generalization $\mathcal{L} R(r, 0, \beta) \equiv \Delta G(r, 0,-E)$ of Eq. (14), we have thought it of interest to plot the righthand side of this equation for positive $E$ in Fig. 2 for $Z=1$. For negative $E$, a slightly different formula is needed, namely,

$$
\mathcal{L} R(0,0, \beta)=-\frac{Z \gamma}{\pi}-\frac{Z}{\pi} \Psi\left(1+\frac{i Z}{\sqrt{-2 E}}\right) .
$$

We have not to date succeeded in inverting the Laplace transform in Eq. (14) by purely analytic means, but progress has proved possible by combining the scaling properties of the quantity $R(0,0, \beta)$, in which of course the dependence on atomic number $Z$ is implicit, with a formula for the inverse Laplace transform due to Widders [6]. Taking first the scaling property of $R(0,0, \beta)$ given by Eq. (8), it is a straightforward matter to demonstrate that

$$
R(0,0, \beta)=\frac{1}{(2 \pi \beta)^{3 / 2}} F\left(Z^{2} \beta\right) .
$$

Our aim below is then to determine the function $F(x)$ introduced in Eq. (17) using the result of Widders for the inverse Laplace transform of a function $f(s)$, namely,

$$
\mathcal{L}^{-1} f(s)=\left.\lim _{n \rightarrow \infty}\left[\frac{(-1)^{n}}{n !}\right] s^{n+1} f^{(n)}(s)\right|_{s=n / t} .
$$

While we are not able so far to use Eq. (14) exactly, we have verified that, to graphical accuracy, one can evaluate Eq. (18) to $n=200$, and the result thus obtained numerically 

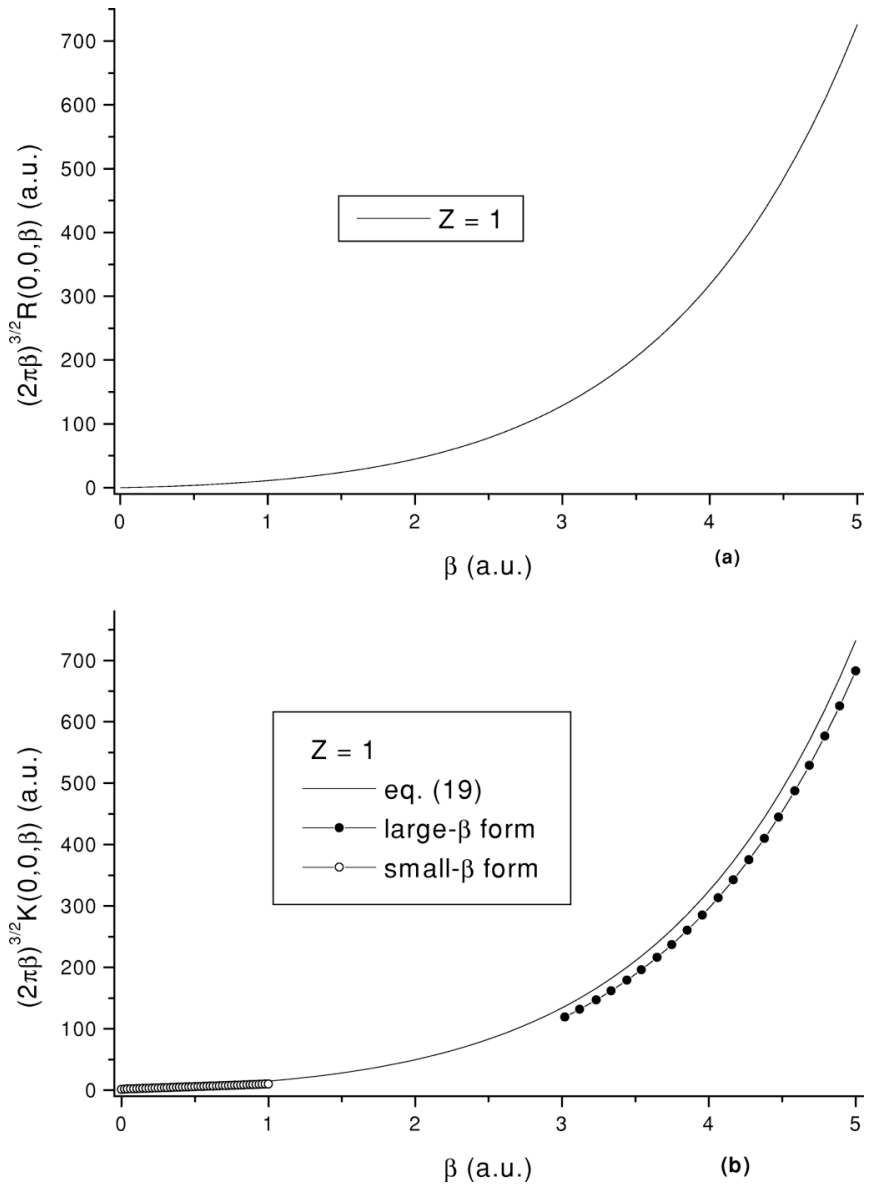

FIG. 3. (a) The quantity $(2 \pi \beta)^{3 / 2} R(0,0, \beta)$ for $Z=1$, evaluated using Widder's formula for the inverse Laplace tranform of Eq. (14), with $n=200$; (b) $(2 \pi \beta)^{3 / 2} K(0,0, \beta)$ for $Z=1$, evaluated from Eq. (19). The small- $\beta$ and large- $\beta$ approximate forms of Eqs. (20) and (23) at $r=0$ are also shown for comparison.

for $(2 \pi \beta)^{3 / 2} R(0,0, \beta)$ is plotted as a function of $\beta$ in Fig. 3(a). But Eqs. (6) and (7) allow $K(0,0, \beta)$ to be determined from

$$
K(0,0, \beta)=\frac{1}{(2 \pi \beta)^{3 / 2}}+\frac{Z}{2 \pi \beta}+R(0,0, \beta),
$$

and using Fig. 3(a), $(2 \pi \beta)^{3 / 2} K(0,0, \beta)$ is shown as a function of $\beta$ in Fig. 3(b). For comparison, we show also in Fig. 3(b) both small $x$ and large $x$ analytical forms of $(2 \pi \beta)^{3 / 2} K(0,0, \beta)$. Specifically, the small- $x$ form is readily shown from the work of Blinder [1] to be given by

$$
\begin{aligned}
(2 \pi \beta)^{3 / 2} K(0,0, \beta)= & +(2 \pi \beta)^{1 / 2} Z+4 \sqrt{\pi} \frac{\zeta(2)}{\Gamma(1 / 2)}\left(\frac{x}{2}\right) \\
& +4 \sqrt{\pi} \frac{\zeta(3)}{\Gamma(1)}\left(\frac{x}{2}\right)^{3 / 2}+\cdots
\end{aligned}
$$

where $\zeta(n)$ is the Riemann zeta function. As to the large- $x$ form, one knows that for sufficiently large $\beta$ the Feynman propagator with $\beta=i t$ is dominated by the lowest bound state with wave function

$$
\psi_{1 s}(r)=\left(\frac{Z^{3}}{\pi}\right)^{1 / 2} \exp (-Z r)
$$

which yields almost immediately, since the bound-state energy $\epsilon_{0}=-Z^{2} / 2$,

$$
K(r, 0, \beta)_{\beta \rightarrow \infty}=\left(\frac{Z^{3}}{\pi}\right)^{1 / 2} \psi_{1 s}(r) \exp \left(\frac{Z^{2} \beta}{2}\right),
$$

and hence

$$
(2 \pi \beta)^{3 / 2} K(r, 0, \beta)_{\beta \rightarrow \infty}=\exp \left(-Z r+Z^{2} \beta / 2\right) \frac{\left(2 \pi \beta Z^{2}\right)^{3 / 2}}{\pi} .
$$

This limiting form (23) is also plotted at $r=0$ as a function of $\beta$ in Fig. 3(b) and is in satisfactory accord with the approximation in which the $n \rightarrow \infty$ in the formula (18) is replaced by $n=200$. We conclude therefore that $K(0,0, \beta)$ is known to satisfactory numerical accuracy for all values of $x=Z^{2} \beta$.

From the investigation of March and Murray [3,4], we have the integral equation for $K(\mathbf{r}, 0, \beta)$ as

$$
\begin{aligned}
K(r, 0, \beta)= & K_{0}(r, 0, \beta)+\int d \mathbf{r}_{1} \int_{0}^{\beta} d \beta_{1} K_{0}\left(\mathbf{r}, \mathbf{r}_{1}, \beta-\beta_{1}\right) \\
& \times \frac{Z}{r_{1}} K\left(\mathbf{r}_{1}, 0, \beta_{1}\right)
\end{aligned}
$$

or

$\mathcal{L} K(r, 0, \beta)=\mathcal{L} K_{0}(r, 0, \beta)+\int d \mathbf{r}_{1} \mathcal{L} K_{0}\left(\mathbf{r}, \mathbf{r}_{1}, \beta\right) \frac{Z}{r_{1}} \mathcal{L} K\left(\mathbf{r}_{1}, 0, \beta\right)$.

This is equivalent to the integral equation for $G(r, 0,-E)$ :

$$
\begin{aligned}
G(r, 0,-E)= & \frac{\exp (-\sqrt{2 E} r)}{r}+\int \frac{\exp \left(-\sqrt{2 E}\left|\mathbf{r}-\mathbf{r}_{1}\right|\right)}{\left|\mathbf{r}-\mathbf{r}_{1}\right|} \frac{Z}{r_{1}} \\
& \times G\left(\mathbf{r}_{1}, 0,-E\right) d \mathbf{r}_{1} .
\end{aligned}
$$

Since the recent paper by Chibisov et al. [7] is also concerned with the Coulomb Green function, it seems appropriate to establish contact between their work and our own studies. First, we note the spatial generalization of Kato's theorem by one of us [8], namely,

$$
\frac{\partial \rho}{\partial r}=-\frac{2 Z}{a_{0}} \rho_{s}(r),
$$

where $Z$ is the atomic number, $a_{0}$ is the Bohr radius $\hbar^{2} / m e^{2}$, while $\rho_{s}(r)$ is the "s-state $(l=0)$ only" contribution to the total density $\rho(r)$, Eq. (27) being valid for an arbitrary number of closed shells. In passing, we note that Eq. (15) of Chibisov et al. [7] is a special case of Eq. (27) applying to a single closed shell of principal quantum number $n$. Summing their Eq. (15) over an arbitrary number of closed shells leads directly to Eq. (27). 
However, the most important result of Chibisov et al. [7], as indeed they stress, which follows from their Green's function analysis, is their Eq. (10) which reads, in the present context,

$$
\begin{aligned}
\gamma_{n}(\mathbf{r}, \mathbf{R}) & =\frac{4 Z_{n}\left[\phi_{n 0}^{\prime}\left(Z_{n} y\right) \phi_{n 0}\left(Z_{n} x\right)-\phi_{n 0}\left(Z_{n} y\right) \phi_{n 0}^{\prime}\left(Z_{n} x\right)\right]}{(x-y)}, \\
x & =r+R+|\mathbf{r}-\mathbf{R}|, \quad y=r+R-|\mathbf{r}-\mathbf{R}| .
\end{aligned}
$$

This implies that the Dirac density matrix for the $n$th closed shell is itself uniquely determined by the $s$-state density $\rho_{n s}(r)$, or alternatively by its $s$-state wave function $\psi_{n 00}(r)$ $=\phi_{n 0}(r) / r=\rho_{n s}^{1 / 2}(r)$. Whereas we have not presently been able to sum Eq. (28) over $n$ to get $\gamma\left(\mathbf{r}, \mathbf{r}^{\prime}\right)$, the result of Komarov [9] (and independently of Theophilou and March [10]), which is valid for an arbitrary number of closed shells for the total Dirac matrix, and reads

$$
\gamma(\mathbf{r}, \mathbf{R})=-\frac{1}{(x-y)}\left(\frac{\partial}{\partial x}-\frac{\partial}{\partial y}\right)\left[x y \gamma_{s}\left(\frac{x}{2}, \frac{y}{2}\right)\right],
$$

shows that, since

$$
\gamma_{s}(r, R) \equiv \sum_{\text {closed shells }} \gamma_{n s}(r, R),
$$

the $s$-state density matrix determines the total matrix $\gamma(\mathbf{r}, \mathbf{R})$. Unfortunately, to date we have not succeeded in finding a closed form for $\gamma_{s}(r, R)$ for an arbitrary number of closed shells. The obvious merit of Eq. (10) of Chibisov et al. [7] is that only the diagonal density $\rho_{n s}(r)$ is needed to determine $\gamma_{n}(\mathbf{r}, \mathbf{R})$, i.e., the total Dirac matrix of the $n$th closed shell.

In the above context, Heilmann and Lieb [11] have summed the squares of the hydrogenic wave functions $\psi_{n l m}(\mathbf{r})$ over $l$ and $m$ and then over $n$ from one to infinity, i.e., over the entire bound-state spectrum. It would be of interest, we feel, if the $n$th shell Dirac matrix in Eq. (10) of Chibisov et al. [7] had a limit as $n \rightarrow \infty$, but so far we have not been able to prove, or disprove, such a conjecture. However, the properties of the particular Whittaker function $W_{n}$ as $n \rightarrow \infty$ (see Eq. (8) of Ref. [7]) seem to hold the key to establish, or disprove, this speculation.

In summary, the main results obtained here are embodied in Eqs. (6) and (14), which have led us to construct Figs. 1-3 above. We have also established the relation between the study of Chibisov et al. [7] and the spatial generalization of Kato's theorem [8] set out in Eq. (27) above.

We are most grateful to Professor I.V. Komarov for invaluable correspondence which has certainly influenced considerably the presentation of this work. I.A.H. acknowledges support from the IWT-Flemish region under Grant No. IWT161. N.H.M. wishes to acknowledge partial financial support for work on density-functional theory from the ONR. Dr. P. Schmidt of that Office is especially thanked for much motivation and his continuing support.
[1] S. M. Blinder, Phys. Rev. A 43, 13 (1991).

[2] N. H. March and A. M. Murray, Phys. Rev. 120, 830 (1960).

[3] N. H. March and A. M. Murray, Proc. R. Soc. London, Ser. A 261, 119 (1961).

[4] For a summary, see N. H. March, W. H. Young, and S. Sampanthar, The Many-Body Problem in Quantum Mechanics (Dover, New York, 1995).

[5] M. Abramowitz and I. A. Stegun, Handbook of Mathematical Functions (Dover, New York, 1970).

[6] See, for example, C. L. Bohn and R. W. Flynn, Am. J. Phys.
46, 1250 (1978).

[7] M. Chibisov, A. M. Ermolaev, F. Brouillard, and M. H. Cherkani, Phys. Rev. Lett. 84, 451 (2000).

[8] N. H. March, Phys. Rev. A 33, 88 (1986).

[9] I. V. Komarov, Diploma thesis, Leningrad State University, 1964 (unpublished) (in Russian).

[10] A. K. Theophilou and N. H. March, Phys. Rev. A 34, 3630 (1986).

[11] O. J. Heilmann and E. H. Lieb, Phys. Rev. A 52, 3628 (1995). 